the American Mathematical Society vol. 3 (1952) pp. 821-828.

2. Nils Sjöberg, Sur les minorantes sousharmoniques d'une fonction donnée, Proceedings of the Ninth Scandanavian Mathematical Congress, Helsingfors, 1938, pp. 309-319.

3. Marcel Brelot, Minorantes sous-harmoniques, extrémales et capacites, J. Math. Pures Appl. (9) vol. 24 (1945) pp. 1-32.

4. E. Szpilrajn, Remarques sur les fonctions sousharmoniques, Ann. of Math. vol. 34 (1933) pp. 588-594.

5. T. Rad6, Subharmonic functions, Ergebnisse der Mathematik und ihrer Grenzgebiete, Berlin, 1937.

University of California, Los ANgeles, AND INSTITUTE FOR ADVANCED STUDY

\title{
LATTICE ORDERING ON BANACH SPACES ${ }^{1}$
}

\section{J. HEIDER}

In all that follows the letter $B$ will denote a given real Banach space, the letter $X$ a bicompact Hausdorff space, and the symbol $C(X)$ the space of all real-valued continuous functions $c(x)$ on the space $X$. The space $C(X)$ may be considered, among other ways, as a real Banach space under the norm $\|c(x)\|=1$.u.b. $|c(x)|, x \in X$, and as a linear lattice under the partial ordering $c_{1}(x) \geqq c_{2}(x)$ for $c_{1}(x)$ $-c_{2}(x)$ non-negative on $X$.

A space $B$ equivalent to a $C(X)$ under a linear norm-preserving isomorphism is, by transfer of the natural order on $C(X)$, susceptive of a partial ordering under which $B$ becomes both equivalent and lattice-isomorphic to $C(X)$. Thus the question of the purely Banachspace characterization of $C(X)$ is identical with the question of the linear normed lattice characterization of $C(X)$ provided the necessary and sufficient conditions under which a given Banach space is susceptible to a linear lattice ordering together with the additional conditions of the linear lattice characterizations are expressible in terms meaningful in all Banach spaces. This principle, applied in reference to the Kakutani [3] ${ }^{2}$ linear normed lattice characterization of $C(X)$, is implicit in the Clarkson [2] Banach space characterization of $C(X)$. The purpose of this note is to present the methods

Received by the editors January 19, 1952.

1 This note constitutes part of a thesis submitted to the University of Michigan. The author is grateful to Professor S. B. Myers for his advice.

2 Numbers in brackets refer to the references at the end of the paper. 
of Clarkson in their full generality, to express the simplicity of these results when reference is made to the Krein [5] rather than to the Kakutani characterization, and, lastly, to apply these principles to a characterization of those Banach spaces which are susceptive of a partial ordering under which they become the abstract $(L)$ spaces of Kakutani.

1. A relation $\geqq$ will be said to provide a linear partial ordering of space $B$ if it satisfies the conditions:

( $\alpha$ ) $b \in B$ implies $b \geqq b$.

( $\beta) \quad b \geqq c$ and $c \geqq b$ imply $b=c$.

( $\gamma) b \geqq c$ and $c \geqq d$ imply $b \geqq d$.

( $\delta) b \geqq c$ implies $b+d \geqq c+d$ and $m b \geqq m c$ for all $d \in B$ and all scalars $m \geqq 0$.

It is known $[1$, p. 214] that space $B$ or any linear space is susceptive of such a linear partial ordering if and only if $B$ contains a subset $B^{+}$ with properties:

(A) $0 \in B^{+}$.

( $\mu$ ) $b, c \in B^{+}$imply $b+c \in B^{+}$and if $b+c=0$, then $b=0=c$.

(v) $b \in B^{+}$implies $m b \in B^{+}$for all scalars $m \geqq 0$.

Assume that $B$ contains a subset $B^{+}$with these properties and consider $B$ under the corresponding linear partial ordering. It is known $[1$, p. 215] that $B$ will be a linear lattice if and only if the element $b^{+}=b \vee 0$ exists in the usual sense for every $b \in B$. This fact simplifies the proof of the following:

Lemma 1.1. Space $B$ (or any linear space) with subset $B^{+}$is a linear lattice if and only if $b \in B$ implies there exists $b^{+} \in B$ such that $\left(B^{+}+b\right)$ $\cap B^{+}=\left(B^{+}+b^{+}\right)$.

Proof. Assume that $b \bigvee 0=b^{+}$exists in the lattice sense for a particular $b \in B$. Then $\left(B^{+}+b^{+}\right) \subseteq\left[\left(B^{+}+b\right) \cap B^{+}\right]$since for arbitrary $b_{0}^{+}$in $B^{+}$also $b_{0}^{+}+b^{+}$is in $B^{+}$while $b_{0}^{+}+b^{+}=\left(b_{0}^{+}+b^{+}-b\right)+b=b_{1}^{+}+b$ with $b_{1}^{+}$in $B^{+}$(since $b^{+} \geqq b$ implies $b^{+}-b$ is in $B^{+}$, so that $b_{0}^{+}+\left(b^{+}-b\right)$ $=b_{1}^{+}$is in $\left.B^{+}\right)$. Likewise $\left[\left(B^{+}+b\right) \cap B^{+}\right] \subseteq\left(B^{+}+b^{+}\right)$, for if $b_{1}^{+}+b=b_{2}^{+}$, then $b_{2}^{+}-b=b_{1}^{+}$implies $b_{2}^{+} \geqq b$ and $b_{2}^{+} \geqq 0$ so that $b_{2}^{+} \geqq b^{+}$or $b_{2}^{+}-b^{+}$ $=b_{3}{ }^{+}$. Thus $b_{1}^{+}+b=b_{2}^{+}=b_{3}{ }^{+}+b^{+}$with $b_{3}{ }^{+}$in $B^{+}$. Hence the existence of $b \bigvee 0$ implies $\left(B^{+}+b\right) \cap B^{+}=\left(B^{+}+[b \bigvee 0]\right)$. Conversely, assume that for a particular $b \in B$ there exists $b^{+} \in B$ such that $\left(B^{+}+b\right) \cap B^{+}$ $=\left(B^{+}+b^{+}\right)$. Then $0 \in B^{+}$implies $b^{+} \in B^{+}$so that $b^{+} \geqq 0$. Also there exists $b_{1}^{+} \in B^{+}$such that $b_{1}^{+}+b=b^{+}=0+b^{+}$. Hence $b^{+}-b=b_{1}^{+}$or $b^{+} \geqq b$. Finally assume there exists $b_{0}^{+}$with $b_{0}^{+} \geqq 0$ and $b_{0}^{+} \geqq b$. Then $b_{0}^{+}-b=b_{1}^{+}$or $b_{1}^{+}+b=b_{0}^{+}$implies there exists $b_{2}^{+}$such that $b_{1}^{+}+b$ 
$=b_{0}^{+}=b_{2}^{+}+b^{+}$so that $b_{0}^{+}-b^{+}=b_{2}^{+}$or $b_{0}^{+} \geqq b^{+}$. Hence $b^{+}=b \bigvee 0$ in the usual sense that the equivalence is established.

Henceforth denote by $B^{+\vee}$ any subset of space $B$ founding a linear partial order under which $B$ is a linear lattice. Now in the linear normed lattice $C(X)$ the ordering introduced by the set $C(X)^{+\vee}$ of non-negative functions is related to the norm on $C(X)$ through the element $e(x) \equiv 1,\|e\|=1$, in the following manner: for $c(x) \in C(X)$ one has $\|c(x)\| \leqq 1$ if and only if $-e(x) \leqq c(x) \leqq e(x)$, or, equivalently, both $e(x)+c(x)$ and $e(x)-c(x)$ are in $C(X)^{+\vee}$. Henceforth any subset $B^{+\vee}$ of space $B$ containing in addition an element $e,\|e\|=1$, such that for $b \in B$ one has $\|b\| \leqq 1$ if and only if $-e \leqq b \leqq e$, or, equivalently, both $e+b$ and $e-b$ are in $B^{+\vee}$ will be denoted by the symbol $B^{+\Lambda_{e}}$. We note that the set $B^{+V_{e}}$ is defined in terms meaningful in all Banach spaces.

Thus Banach space $B$ with subset $B^{+V_{0}}$ is susceptive of a partial ordering $\geqq$ such that:

$\left(\mathrm{K}_{1}\right) b \geqq b ; b \geqq c$ and $c \geqq b$ imply $b=c ; b \geqq c$ and $c \geqq d$ imply $b \geqq d$; $b \geqq c$ implies $b+d \geqq c+d$ for all $d \in B$ and $m b \geqq m c$ for all real scalars $m \geqq 0$.

$\left(\mathrm{K}_{2}\right) b \in B$ implies there exists $b^{+} \in B$ such that $b^{+}=b \vee 0$, i.e., $b^{+} \geqq 0$ and $b^{+} \geqq b$ while $c \geqq 0$ and $c \geqq b$ imply $c \geqq b^{+}$.

$\left(\mathrm{K}_{3}\right)$ There exists element $e \in B,\|e\|=1, e>0$, such that for $b \in B$, $\|b\| \leqq 1$ if and only if $-e \leqq b \leqq e$.

It is seen, conversely, that any space $B$ with element $e$ and partial ordering $\geqq$ satisfying these conditions contains a set $B^{+V_{e}}$. However, the conditions $\mathrm{K}_{i}, i=1,2,3$, express, equivalently, the Krein linear normed lattice characterization of $C(X)$. Thus since space $B$ is equivalent to a $C(X)$ if and only if it is susceptive of a linear partial ordering under which it is both equivalent and lattice isomorphic to $C(X)$ and thus satisfies the Krein characterization, we have the following result:

Theorem 1.2. Banach space $B$ is equivalent to a $C(X)$ if and only if $B$ contains a $B^{+V_{e}}$ subset.

2. The preceding number reduced the Banach space characterization to the Krein linear normed lattice characterization. Since the details of the latter are less available than those of Kakutani's characterization, we give a direct proof of their equivalence and show in the process that one postulate in Kakutani's definition of an abstract $(M)$ space with strong unit is redundant.

Lemma 2.1. Space $B$ with relation $\geqq$ satisfying conditions $\mathrm{K}_{1}$ and $\mathrm{K}_{2}$ also satisfies $\mathrm{K}_{3}$ if and only if: 
$\left(\mathrm{K}_{3}^{\prime}\right)$ ( $\left.\rho\right)$ There exists element $e \in B,\|e\|=1, e>0$, such that $\|b\| \leqq 1$ implies $-e \leqq b \leqq e$.

( $\sigma) b \geqq 0, c \geqq 0$ imply $\|b \vee c\|=\max (\|b\|,\|c\|)$.

( $\tau) b \wedge c=0$ implies $\|b+c\|=\|b\| \vdash c \|$.

Proof. Assume that space $B$ satisfies conditions $K_{1}, K_{2}, K_{3}$, and let $b \geqq 0, c \geqq 0$ be elements of $B$ with $\|b\| \geqq\|c\| \geqq 0$. Since $0 \leqq b \leqq b \vee c$ $\leqq\|b \bigvee c\| e$, we have $0 \leqq\|c\| \leqq\|b\| \leqq\|b \vee c\|$. But $0 \leqq b \leqq\|b\| e$ and $0 \leqq c$ $\leqq\|\| e \leqq\|b\| e$ imply $0 \leqq b \vee c \leqq\|b\| e$ so that $\|b \vee c\| \leqq\|b\|$ and hence $\|b \vee c\|=\|b\|=\max (\|b\|,\|c\|)$ and condition $\mathrm{K}_{3}^{\prime}:(\sigma)$ is satisfied.

Next let $b \wedge c=0$ so that $b \geqq 0$ and $c \geqq 0$ and assume $\|b\| \geqq\|c\| \geqq 0$. As in all linear lattices $b+c=b \vee c+b \wedge c$ so that, in the present case, $b+c=b \vee c$. Thus $\|b\|=\|b \vee c\|=\|b+c\|$. Also $2 b=(b-c)+(b+c)$ and $2\|b\| \leqq\|b-c\|+\|b+c\|$ so that $\|b\| \leqq\|b-c\|$. However $\|b\| e \geqq b \geqq b$ $-c \geqq-c \geqq-\|c\| e \geqq-\|b\| e$. Thus $\|b-c\| \leqq\|b\|$ so that $\|b-c\|=\|b\|$ $=\|b+c\|$ and condition $\mathrm{K}_{3}^{\prime}:(\tau)$ follows.

Conversely, assume that space $B$ satisfies conditions $K_{1}, K_{2}, K_{3}^{\prime}$. As in all linear lattices $b=b \vee 0-(-b \vee 0)=b^{+}-b^{-}$with $b^{+} \wedge b^{-}=0$ so that $\|b\|=\left\|b^{+}-b^{-}\right\|=\left\|b^{+}+b^{-}\right\|=\left\|b^{+} \vee b^{-}\right\|=\max \left(\|b\|+,\left\|b^{-}\right\|\right)$. But if $-e \leqq b \leqq e$, then $0 \leqq b \vee 0=b^{+} \leqq e$ and $0 \leqq-b \vee 0=b^{-} \leqq e$ so that $1=\|e\|=\left\|e \vee b^{+}\right\|=\max \quad\left(\|e\|,\left\|b^{+}\right\|\right)$and $1=\|e\|=\left\|e \vee b^{-}\right\|$ $=\max \left(\|e\|,\left\|b^{-}\right\|\right)$. Thus $\|b\|=\max \left(\left\|b^{+}\right\|,\left\|b^{-}\right\|\right) \leqq 1$ and condition $\mathrm{K}_{3}$ is satisfied, and the lemma is proved.

Assume now that space $B$ satisfies conditions $\mathrm{K}_{1}, \mathrm{~K}_{2}$ and $\mathrm{K}_{3}$ or $\mathrm{K}_{3}^{\prime}$. This space will then also satisfy condition:

$\left(\mathrm{K}_{4}\right) \quad b_{n} \geqq 0$ with $\lim _{n}\left\|b_{n}-b\right\|=0$ implies $b \geqq 0$.

Thus, since $\lim _{n}\left\|b_{n}-b\right\|=0$, there exists $K=1$.u.b. $\left(\left\|b_{n}\right\|\right)$. Hence $0 \leqq b_{n} \leqq\left\|b_{n}\right\| e \leqq K e$ for all $n$ or $0 \leqq b_{n} / K \leqq e$ and $-e \leqq b_{n} / K-e \leqq 0$ so that $\left\|b_{n} / K-e\right\| \leqq 1$ for all $n$. But $\lim _{n}\left\|1 / K \cdot\left(b_{n}-b\right)\right\|=0$ so that $\|b / K-e\| \leqq 1$ and $b \geqq 0$.

To summarize, it was shown that for space $B$ with relation $\geqq$ conditions $K_{1}, K_{2}, K_{3}$ are equivalent to conditions $K_{1}, K_{2}, K_{3}^{\prime}$ and that in either case condition $K_{4}$ is satisfied. Moreover the set $B^{+V_{e}}$ is seen to be closed. However space $B$ with relation $\geqq$ satisfying conditions $\mathrm{K}_{1}, \mathrm{~K}_{2}, \mathrm{~K}_{3}^{\prime}, \mathrm{K}_{4}$ is by definition the abstract $(M)$ space with strong unit in terms of which the Kakutani linear normed lattice characterization of $C(X)$ is given. The following result is now evident:

Theorem 2.2. A necessary and sufficient condition that space $B$ be susceptive of a relation $\geqq$ under which it becomes an abstract $(M)$ space with strong unit is that it contain a $B^{+V_{e}}$ subset.

3. Associated with abstract $(M)$ spaces are what Kakutani calls abstract $(L)$ spaces $[3 ; 4]$ : 
Definition. An abstract $(L)$ space is a space $B$ with a linear partial ordering $\geqq$ such that under this ordering:

$\left(\mathrm{L}_{1}\right) B$ is a linear lattice.

(L) $b_{n} \geqq 0$ with $\lim _{n}\left\|b_{n}-b\right\|=0$ implies $b \geqq 0$.

$\left(\mathrm{L}_{3}\right) b \wedge c=0$ implies $\|b+c\|=\|b-c\|$.

$\left(\mathrm{L}_{4}\right) b \geqq 0, c \geqq 0$ imply $\|b+c\|=\|b\|+\|c\|$.

A purely Banach space characterization of those spaces $B$ which are susceptive of a linear partial ordering under which they become abstract $(L)$ spaces is now possible. Thus assume that $B$ is susceptive of such an ordering. In view of $\mathrm{L}_{1}$ and $\mathrm{L}_{2}, B$ must contain a closed $B^{+\vee}$ subset. Now recall the Myers' [6] definition of a $T$-set, $T$, as a subset of $B$ maximal with respect to the property: $b_{i} \in T, i=1, \cdots, n$, implies $\left\|\sum_{i=1}^{n} b_{i}\right\|=\sum_{i=1}^{n}\left\|b_{i}\right\|$. In view of condition $\mathrm{L}_{4}$ and since the sum of any finite number of non-negative elements is again such an element, it follows that $B^{+\vee}$ is contained in some $T$-set, $T$, of $B$. Now assume $b \in\left(T-B^{+\vee}\right)$. Since $B$ is a linear lattice, $b=(b \vee 0)$ $-(-b \vee 0)=b^{+}-b^{-}$with $b^{+} \wedge b^{-}=0$ and $b^{+}, b^{-}$in $B^{+\vee}$. However $b^{+} \neq 0, b^{-} \neq 0$ is excluded, since otherwise, with $b^{ \pm} \in B^{+\vee} \subseteq T,\left\|b+b^{-}\right\|$ $=\left\|b^{+}-b^{-}+b^{-}\right\|=\left\|b^{+}\right\|<\left\|b^{+}\right\|+2\left\|b^{-}\right\|=\left\|b^{+}+b^{-}\right\|+\left\|b^{-}\right\|=\left\|b^{+}-b^{-}\right\|$ $+\left\|b^{-}\right\|=\|b\|+\| b^{-}$. Also $b^{+}=0, b^{-} \neq 0$ is excluded since, with $b^{-}$ $\in B^{+\vee} \subseteq T,\left\|b+b^{-}\right\|=\left\|-b^{-}+b-\right\|=0<\|b\|+\|b-\|$. The only remaining possibilities are that $b=b^{+}$or $b=0$ so that $b$ is in $B^{+\vee}$ and $T=B^{+\vee}$. Thus any abstract $(L)$ space contains a closed $T$-set, $T$, with the properties corresponding to $\mathrm{L}_{1}$ and $\mathrm{L}_{3}$, that $(T+b) \cap T=\left(T+b^{+}\right)$for some $b^{+}$, while $(T-b) \bigcap(T-c)=T$ implies $\|b-c\|=\|b+c\|$. Denote such a $T$-set by the symbol $T^{\vee \wedge}$.

Conversely, assume that space $B$ contains a $T$-set, $T^{\vee \wedge}$, which is closed. Since every $T$-set, $T$, has the properties:

( $\lambda$ ) $0 \in T$;

( $\mu$ ) $t_{1}, t_{2}$ in $T$ imply $\left(t_{1}+t_{2}\right)$ in $T$, while $t_{1}+t_{2}=0$ implies $t_{1}=0=t_{2}$ since $\left\|t_{1}+t_{2}\right\|=\left\|t_{1}\right\|+\left\|t_{2}\right\|$

( $\nu) t \in T$ implies $m t \in T$ for all scalars $m \geqq 0$;

it follows that space $B$ is susceptive of a linear partial ordering under the relation: $b \geqq c$ if and only if $(b-c) \in T^{\vee \wedge}$, and that under this ordering $B$ becomes an abstract $(L)$ space. Thus:

Theorem 3.1. A necessary and sufficient condition that space $B$ be susceptive of a linear partial ordering under which it becomes an $a b$ stract $(L)$ space is that it contain a closed $T$-set, $T^{\vee \wedge}$.

It is now possible to give purely Banach space interpretations to the work of Kakutani on abstract $(L)$ spaces. In particular:

TheOREM 3.2. If space $B$ contains a close $T$-set, $T^{\vee \wedge}$, then the odd- 
numbered adjoint spaces of $B$ each contain a (closed) set $B^{+V_{e}}$. If space $B$ contains $a$ set $B^{+V_{e}}$, then the odd-numbered adjoint spaces each contain a set $T^{\vee \wedge}$.

This proposition restates the Theorem $15[3$, p. 1021] and the Remark 3 [3, p. 1023] of Kakutani.

\section{BIBLIOGRAPHY}

1. G. Birkhoff, Lattice theory, Amer. Math. Soc. Colloquium Publications, vol. 25, rev. ed., 1948.

2. J. A. Clarkson, A characterization of C-spaces, Ann. of Math. vol. 48 (1947) pp. 845-850.

3. S. Kakutani, Concrete representations of abstract ( $M$ ) spaces, Ann. of Math. vol. 42 (1941) pp. 994-1024.

4. - Concrete representation of abstract $(L)$ spaces and the mean ergodic theorem, Ann. of Math. vol. 42 (1941) pp. 523-537.

5. M. and S. Krein, On an inner characteristic of the set of all continuous functions defined on a bicompact Hausdorf space, C. R. (Doklady) Acad. Sci. URSS. vol. 27 (1940) pp. 427-430.

6. S. B. Myers, Banach spaces of continuous functions, Ann. of Math. vol. 49 (1948) pp. 132-140.

UNIVERSITY OF MichigAN 